\title{
Análisis del Trabajo en las Prácticas de Auditoría, en las Empresas de Servicios Públicos, en Lima Metropolitana
}

Analysis of Work in Practice Audit in

Public Services Companies in Lima

Félix Armando Rivera León *

felix_arl@hotmail.com

[RECEPCIÓN: AGOSTO 2016 / CONFORMIDAD: OCTUBRE 2016]

\begin{abstract}
RESUMEN
La investigación se encuentra orientada a buscar las causas o razones que generalmente contribuyen a la falta de calidad en los resultados del trabajo del auditor, cuya responsabilidad es de los auditores responsables, supervisores o auditor interno, que firman el informe final.

Del mismo modo, se incide en cuanto a la eficiencia en los trabajos de auditoría, así como en el fomentar, inculcar y defender la independencia de criterio, el resaltar que las entidades empresariales, como las sociedades de auditoría, deben obligarse a cumplir las normas internacionales de auditoría, y otras que demanden su responsabilidad profesional.
\end{abstract}

Palabras clave: Prácticas, auditoría, empresas públicas

\section{ABSTRACT}

This research is guided to look for the causes or reasons that generally contribute to the lack of quality in the results of the auditor's work whose responsibility belongs to the responsible auditors, supervisors or internal auditor that sign the final report.

It has a bearing on the efficiency in the audit works as well as in developing, instilling, and defending the approach independence, standing out that the managerial entities, as the audit societies should put under an obligation to complete the international norms of audit, and others that demand their professional responsibility.

Key words: Audit practices service companies

\footnotetext{
Doctor en Ciencias Contables y Empresariales por la Universidad Nacional Mayor de San Marcos (UNMSM), Magíster en Administración con mención en Gestión Empresarial(UNMSM), Contador Público Colegiado, Profesor Principal de la Facultad de Ciencias Administrativas (UNMSM).
} 


\section{INTRODUCCIÓN}

El objetivo de la función de auditoría es aumentar la confianza que se puede tener en la información suministrada en una determinada empresa, donde tal confianza puede definirse como congruencia entre el mensaje transmitido y la realidad que se describe. Por lo tanto, la auditoría puede tener una revisión de la información por una persona ajena a quien la preparó, lo cual hace que aumente la probabilidad de que refleje con exactitud lo que tiene de pretensión de dar a conocer. Este contexto involucra aspectos estrictamente financieros como administrativos.

La importancia de lo expresado se debe fundamentar en las características del profesional a realizar dicha función, que, por lo general, son las siguientes: un conjunto de conocimientos especializados; un proceso educativo formal y reconocido, para adquirir el requisito de conocimiento especializado; una norma de calificaciones profesionales que regulen la admisión a la profesión; reconocimiento de su posición; una norma de conducta que gobierne las relaciones del profesionista con sus clientes, colegas y público; una aceptación de la responsabilidad social inherente a una ocupación que cuenta con interés público; una organización dedicada al avance de las obligaciones sociales de grupo.

Es cierta la necesidad puntualizada de la auditoría, y si esta, en una forma u otra, existe en nuestra patria, reforzada y sustentada a través de las universidades, colegios profesionales, escuelas especializadas, como la Contraloría General de la República, Sociedades de Auditoría, la preocupación deviene como consecuencia de las lecturas de las resoluciones de la Comisión Nacional Supervisora de Empresas y Valores (CONASEV), que sancionan a las sociedades de auditoría por las deficiencias que presentan sus trabajos de auditoría financiera, así como de la lectura de actas de directorio, en las que se enfatizan las discusiones y discrepancias de opinión frente a los informes de auditoría interna, con respecto a la auditoría administrativa. Así, resaltan serios cuestionamientos acerca de la calidad de los mismos; tratando de encontrar posibles causas, se expresan interrogantes como: ¿cuán deficiente es el trabajo del auditor? ¿Del auditor encargado o del auditor responsable? ¿Son aplicadas apropiadamente las normas internacionales de auditoría, en el caso de los estados financieros? ¿Se evalúa apropiadamente la aplicación de los principios de administración en la entidad? ¿Existe negligencia? ¿La supervisión se encuadra dentro de un sistema de control de calidad? ¿La evidencia obtenida es suficiente y competente? ¿Cuál es la percepción ético-moral que tienen los clientes de los auditores?

La investigación consistió en analizar la actuación del trabajo profesional del auditor, es decir, "la diligencia debida"; estudiar sobre "la deficiencia de los trabajos de auditoría", cuya responsabilidad de actuación es de los auditores responsables (supervisores), que firman el informe, o del auditor interno; asimismo, la investigación trató de evaluar el "descrédito de los auditores" a través de la percepción ético-moral de los auditores y la necesidad empresarial de la auditoría. Otro de los puntos preocupantes es la opinión de las empresas comprometidas: se señala las reacciones que han tenido con relación al tema de investigación que realizaremos.

El trabajo se basó en encuestas y cuestionarios a empresas de servicio auditadas en el ámbito de Lima Metropolitana y resultados estadísticos que permiten llegar a criterios concretos y, finalmente, a conclusiones.

La práctica de la auditoría adolece actualmente de algunos problemas inherentes en la evaluación de los sistemas de información, en los cuales se apoyan las gestiones empresariales. Así, uno de los problemas con que se enfrentan las empresas hoy en día es la alta competencia, debido a la apertura económica y otros factores que inciden para que la preocupación de las empresas en cumplir sus objetivos mediante la eficacia, la eficiencia y la economía, es decir, realizando y distribuyendo los recursos de la mejor forma y buscando bajar los costos y gastos; consecuentemente, aumentando las utilidades. Como administradores, somos conscientes de este problema y de lo importante que es la realización de gestiones de control y de resultados con el fin de cumplir los objetivos.

En el contexto mencionado, el auditor dentro del ramo de la información administrativa/financiera desempeña tres papeles fundamentales: verificar la exactitud/ lo correcto de la información, dar a conocer los resultados de sus investigaciones y celebrar sesiones de consulta con la gerencia. El objetivo principal es aumentar la confiabilidad que se puede tener en la información.

La preocupación, motivo de la investigación, se centra en la necesidad de presentar un análisis crí- 
tico, relacionado con la diligencia profesional, los trabajos de auditoría y la percepción ética-moral que deben tener los clientes de los auditores.

Los problemas surgen generalmente como consecuencia del desconocimiento de prácticas, procedimientos mínimos de acción y del manejo auditor, así como de la metodología del proceso de auditoría comprometida de los sistemas de control interno con los sistemas de información gerencial. En el caso de los contadores públicos, está la existencia de normas, procedimientos, metodologías que funcionan dentro de la labor del auditor como paradigmas, y que están obligados a cumplir; en el caso de otras profesiones autorizadas por ley, como el de los administradores, no existen normas de auditoría, generalmente aplicables a los aspectos administrativos, y los que en auditoría lo realizan, lo efectúan sobre la base de sus propios criterios y experiencia.

La interrogante es: el alto riesgo que corre el auditor, como profesional al emitir opinión sobre gestiones administrativas/financieras, así como de resultados económicos, en los que se validan las decisiones gerenciales y sectoriales, que bajo las mencionadas circunstancias de evaluación entraría dentro de un marco de insuficiencia el trabajo realizado por los auditores.

¿Qué causas más comunes generan deficiencias durante el desarrollo del trabajo de los auditores y qué consecuencias generan los resultados del trabajo de los auditores, con relación a la prestación de servicios de auditoría en las empresas de servicio público, en Lima Metropolitana?

El objetivo de la investigación es (1) explicar las causas y consecuencias de la deficiencia en realización de tareas de los auditores, en las empresas de servicio público de Lima Metropolitana, mediante estudios para determinar la adecuada formación profesional del auditor. (2) Analizar las causas por la falta de conocimiento de las prácticas de auditoría (3) Identificar la magnitud de las auditorías deficientes. (4) Precisar y evaluar el grado de deficiencia en los trabajos de auditoría. Y (5) precisar el grado de percepción ético-moral de las empresas comprometidas en el presente estudio.

La contribución de la investigación consiste en establecer criterios que tienen que ver, principalmente, con el objetivo de las prácticas de auditoría y sus resultados, el conocimiento de los elementos auditados y la capacidad de detectar riesgos. La auditoría, tal como se entiende en el ámbito internacional, en los colectivos de profesionales, no tiene como propósito esencial saber si un determinado control, tanto predeterminado como adecuado, se ha implantado simplemente, sino saber qué controló y qué controles existen con la misma finalidad, qué objetivo y qué fines tienen, cómo se realizan, qué eficiencia tienen en cuanto al cumplimiento. y qué riesgos existen aún para los sistemas de información, o bien prejuicios que pueden causar indirectamente.

El sujeto de investigación se encuentra focalizado en las actividades propias del profesional como auditor contable/administrativo. La información empresarial como elemento fuente es esencial para el logro de criterios que fundamenten su opinión, como resultado de su labor, la misma que se encuentra rodeada de riesgos, al tratar de evaluar todas y cada una de la operaciones inmersas en el sistema empresarial, con algunas irregularidades, las que deben estar dentro de límites tolerables. De ahí nuestro estudio intenta realizar y lograr criterios que coadyuven a establecer que la revisión de los elementos de juicio sirva como base para las pruebas de cumplimiento y evaluación del sistema administrativo y/o financiero en las empresas.

La investigación es importante para las sociedades de auditoría, instituciones y empresas en general, ya que permitirá generar un marco laboral conceptual para delinear, en un futuro, estrategias de evaluación que conlleven a metodologías de trabajo de auditoría en todo su contexto integral.

Actualmente, no existen normas generalmente aceptadas en los aspectos de auditoría administrativa que permitan uniformizar criterios de evaluación. Las auditorías que actualmente se realizan del tópico señalado se basan en el criterio individual de los auditores; el estudio se apoya en su experiencia profesional. En lo que respecta al ámbito financiero, nos apoyaremos en la utilización por las sociedades de auditoría, en cuanto a la aplicación de las normas internacionales de auditoría generalmente aceptadas, así como todo aquello que tenga correlación.

\section{MÉTODOS}

Es exploratorio; su alcance temporal se considera actual; su relación práctica es aplicada; su naturaleza es metodológica; su carácter trata sobre causas o efectos; sus fuentes se conside- 
raron primarias. El diseño estuvo constituido por empresas e instituciones del medio, en Lima metropolitana, las mismas que poseen auditorías previamente realizadas. Sin embargo, como no se trata de un estudio que pretenda hacer generalizaciones para todo el universo; sino, más bien, para el logro de las encuestas, bastó trabajar con una población seleccionada y estratificada, que permita condiciones de desarrollo de los resultados. También es inductivo, al efecto se ha considerado en lo posible las siguientes unidades de análisis: actuación de los auditores encargados en ejercicio (no socios), actuación profesional de los servidores responsables de la firma del informe final de auditoría, percepción de los trabajos de auditoría por parte de los directivos (recepcionan el informe de auditoría como producto final).

La realización de la observación será mediante encuestas y cuestionarios previamente confeccionados, con el objeto de que dichos elementos de juicio cumplan una función de enlace entre los objetivos de la investigación y la realidad de la población observada. Por ello, se realizará a través de preguntas concretas sobre dicha realidad, $y$, por otra parte, debe ser capaz de suscitar en los encuestados, respuestas sinceras y claras a cada pregunta, con el objeto de que puedan ser clasificadas y analizadas.

\section{RESULTADOS}

POBLACIÓN: Ocho empresas de servicio público en Lima Metropolitana. Se tomó como referencia en la población a considerar aplicables a los sujetos en investigación a profesionales autorizados a auditar por sus respectivos colegios profesionales, así como referencias de registro de la Contraloría General de la República, asimismo para el caso de las entidades perceptoras de los servicios de auditoría, aquellas que se encuentran obligadas a presentar información financiera. En los casos de la incidencia administrativa, aquellas entidades inscritas al Sistema Nacional de Control, por poseer el Órgano Interno de Control, encargadas de considerar dicho enfoque.

Construcción del instrumento de medición.Para la validación del constructo, en el área de asistencia técnica se contó con 10 personas de distintos sexos y edades pertenecientes a la profesión de contadores y administradores.

Los cuestionarios fueron realizados a los distintos segmentos de auditores y directivos en sus respectivos lugares de trabajo, con una previa solicitud de visita, solicitud de permiso, conocimiento del formato correspondiente.

De esta forma, se pudo interiorizar al encuestado en los fines de la investigación, quienes solicitaron su plena confidencialidad; dado de otro modo, se estaría quebrando las normas personales exigidas a todo auditor.

La encuesta se llevó a cabo en un promedio de 45 a 60 minutos por persona para poder crear ambiente y empatía entre el encuestado y el encuestador, ya que además se deseó saber cuál es la importancia de las distintas dimensiones aplicadas en el SPSS (en los casos de los auditores encargados y supervisores) y el SERVQUAL (directivos), se trasluce en las conversaciones mutuas.

Los cuestionarios han dependido para su confección de la naturaleza de trabajo de cada personaje implicado, utilizado como medida de valor, la tabla Likert, (5 a 1 en forma decreciente); es decir:

1. Para los auditores encargados (220 sujetos), responsables de la elaboración en el campo del desarrollo de los procedimientos, metodologías y cumplir estrictamente las normas inherentes a su especialidad, constan de cuatro partes: la primera dedicada al cumplimiento de las normas técnicas de auditoría; la segunda, a la tenencia y cumplimiento de manuales; la tercera, a la aplicación de programas de auditoría; y, por último, cuestiones sobre aspectos subjetivos, todos ellos conllevan un apropiado desarrollo del trabajo del auditor.

2. Para los supervisores (40 sujetos), responsables de la redacción, firma y emisión del informe final, consta de tres partes: la primera, evaluación de los papeles de trabajo emitidos por los auditores encargados; la segunda, evaluar las evidencias formuladas y presentadas como sustento de los hallazgos sostenidos; y la tercera, respecto a la formulación del informe final de auditoría, producto final de la labor.

- Los directivos (152 sujetos), personas que recepcionan el informe final de auditoría como trabajo terminado por los supervisores; consta de cinco partes:

tangibilidad; fiabilidad; capacidad de respuesta; seguridad; y empatía.

Aspectos que se evalúan bajo la metodología SERVQUAL (Fernández, M 2000). 


\section{Validación del instrumento de medición}

Para la presente investigación se utilizó como instrumento de medición los paquetes computarizados, SPSS (STATISTICAL PACKAGE SOCIAL SCIENCE - PAQUETE ESTADÍSTICO PARA LAS CIENCIAS SOCIALES) y el SERVQUAL (SERVICE QUALITY - SERVICIO DE CALIDAD), aplicados indistintamente a cada uno de los personajes señalados.

El objetivo del análisis de fiabilidad fue determinar que un conjunto de ítems de una escala pueda conducir a resultados que estén altamente correlacionados con los resultados que se llegarían a obtener si se aplicara una prueba que mida lo mismo. Dicho en otra palabras, consiste en lograr una escala que conduzca a resultados similares cuando diferentes personas la administran y cuando se usan formas alternas de la prueba (FERNÁNDEZ, 2000).

Uno de los coeficientes más comunes para calcular la fiabilidad es el Alpha de Cronbach (CRONBACH, 1951), que se orienta hacia la consistencia interna de una prueba y refleja el grado en que convocarían las preguntas que constituyen la escala. Para valores inferiores a 0,6 se considera una baja fiabilidad. Entre 0.6 y 0.8 es aceptable. Por encima de 0.8 es excelente, indicará una alta homogeneidad y equivalencia de respuesta a todos los ítems a la vez y para todos los encuestados (DÍAZ, 2003).

Una vez realizada en su totalidad la encuesta, se procedió al análisis de los datos, con el objetivo de determinar las dimensiones e ítems más representativos $\mathrm{y}$, de esta forma, confirmar cuáles son los factores de desarrollo y calidad en la investigación.

Se dice que un instrumento es fiable cuando con el mismo se obtienen resultados similares al aplicarlo dos o más veces al mismo grupo de individuos o cuando lo que se aplica son formas alternativas del instrumento (VISOUTA, 1998).

\section{HIPÓTESIS HIPÓTESIS (1)}

Las labores del equipo de auditoría carecen de eficiencia y eficacia en los resultados de sus trabajos, que se explican por el desconocimiento o mala aplicación de los criterios normativos y casuísticos que los sustenten.

\section{VARIABLES}

VARIABLE DEPENDIENTE: Diligencia en los procesos casuísticos.

Se considera como variable a los auditores, $y$ su entorno de responsabilidad como elemento de estudio:

Auditores encargados en ejercicio (no socios)

VARIABLES INDEPENDIENTES: Normas, manuales, programas, aspectos subjetivos.

Estas estuvieron implícitas en el siguiente contexto; la gestión administrativa a través del uso adecuado de sistemas de información gerencial; la estructura organizacional que permita la obtención de los objetivos prefijados; los resultados obtenidos; planeamiento y programación de tareas; coordinación y regulación de tareas; supervisión de tareas: racionalización de recursos tecnológicos; control de procedimientos de tareas; equilibrio homeostático en las comunicaciones del equipo de trabajo; empatía.

\section{HIPÓTESIS (2)}

Las labores del equipo de auditoría, a pesar de carecer de eficiencia y eficacia en los resultados de sus trabajos, son aceptadas por el supervisor en la elaboración del informe final como producto.

\section{VARIABLES}

VARIABLE DEPENDIENTE: Calidad en términos de eficiencia y eficacia en el trabajo de los auditores encargados hacia el supervisor.

VARIABLES INDEPENDIENTES: Papeles de trabajo; evidencia; informe de auditoría. Las mismas estuvieron implícitas en el contexto de la variable anterior.

\section{HIPÓTESIS (3)}

El informe final como producto de servicio del equipo de auditores, a pesar de carecer de calidad, es aceptado generalmente por los usuarios (directivos), lo cual se explica por las respuestas en las dimensiones de tangibilidad, fiabilidad, seguridad, capacidad de respuesta y empatía. 


\section{VARIABLES}

VARIABLE DEPENDIENTE: Calidad en el servicio como producto del equipo de auditores (informe final elaborado por los responsables: supervisores). Se considera como variable la calidad en el informe final de los auditores socios o jefe de auditoria interna, que firman el informe (supervisores).

VARIABLE INDEPENDIENTE: Tangibilidad; fiabilidad; seguridad; capacidad de respuesta; empatía

SUJETOS IMPLÍCITOS: Un eficiente trabajo de auditoría puede ser garantizado en la medida que el equipo de trabajo conozca la operatividad y se apoye en una metodología que norme su actuación.

Los niveles de productividad de las instituciones en general podrán mejorar en la medida que las carencias y deficiencias sean detectadas a tiempo a través de una auditoría financiera y/o administrativa oportuna.

La gestión administrativa en las empresas se basa en los objetivos, en los recursos y en el sistema de información implementado.

\section{OPERACIONALIZACIÓN DE LAS VARIABLES}

Después de haber desarrollado las encuestas y presentadas las interpretaciones, se estuvo en condiciones de analizar los resultados a través de comparaciones entre las variables.

Para evaluar la actuación personal y profesional de los auditores no responsables de la forma y contenido del informe (resultado del trabajo), se desarrollaron entrevistas personalizadas, las que permitieron al entrevistador validar la información a nivel de datos en forma objetiva y directa, dirigida a 220 auditores encargados; insertos en C.C.P.L. al 30 de noviembre del 2010, utilizando como instrumento encuestas.

El estudio se dividió en dos fases:

1. EL PROCESO.- Constituido por labores realizadas por los auditores encargados, no poseen responsabilidad final sobre el informe, pero sí ante su respectivo supervisor de trabajo; este último recepciona los trabajos, es su responsabilidad elaborar el informe final de auditoría, sustentándose con información documentaria final de los auditores encargados. Para ello, se ha utilizado SPSS, como soporte de cálculo.
Asimismo, por cada pregunta de las entrevistas realizadas se consideró realizar también como un medio descriptivo: los respectivos estadísticos, sujeto a los auditores mencionados, cuyos comentarios se encuentran al pie de los mismos.

2. LA CALIDAD DEL SERVICIO.- Es el grado que se evalúa a través de los criterios y opiniones de los directivos, quienes reciben el informe final de auditoría, elaborado por el supervisor, cuyo contexto implica la resonancia de los conceptos de expectativa y percepción (SERSQUAL), así como estadísticos descriptivos adicionales.

\section{VALIDEZ TEMÁTICA}

Basado en los conceptos, se ha analizado cuidadosamente su correlación, interpretando la evidencia empírica de acuerdo con el nivel en el que se clarifica la validez del constructo. Para estimar la cuya fiabilidad de las variables, se utilizó Alpha de Cronbach, el cual tiene por objeto determinar si lo que la encuesta pretende determinar está siendo realmente medido a través de dicho instrumento (DÍAZ, 2003).

Se ha utilizado como software de soporte el SPSS, versión 13,0, habiéndose utilizado las pruebas estadísticas de correlación de rangos (Spearman), y estadísticos descriptivos básicos.

\section{RESUMEN DE LOS CASOS PROCESADOS (auditores encargados)}

El modelo Alfa de Cronbach asume que la escala está compuesta por elementos homogéneos aleatoriamente seleccionados de la población de los posibles elementos que miden la misma característica; en el caso de los auditores encargados y sus variables tratadas, existe una fiabilidad, la que determinamos como aceptable, equivalente a: 0.688 , para un número de 12 ítem; incluida la variable diligencia.

\section{AUDITORES ENCARGADOS DIMENSIÓN: NORMAS}

Percepción respecto al trabajo de los auditores encargados de cumplir con las normas técnicas: Los datos indican una percepción similar respecto al desenvolvimiento de los tres auditores encargados de las normas. El indicador apropiado en este caso es la mediana (media 4.91; mediana 5.00 ), que denota una alta valoración respecto al trabajo de los auditores encargados de cumplir 
con las normas técnicas. Esto se ilustra en el siguiente gráfico. Correlación entre percepción del auditor encargado de cumplir con diligencia frente al cumplimiento de las normas técnicas de auditoría: Se observó que en todos los casos existe una correlación positiva, que denota congruencia entre el auditor encargado de cumplir con diligencia la aplicación de las Normas Técnicas de Auditoría. Sin embargo, la medida de esta asociación es en algunos casos débil (0.47 o 0.61) $\mathrm{y}$ solo es fuerte en un caso (rho $=0.80$ ).

\section{AUDITORES ENCARGADOS DIMENSIÓN: LOS MANUALES}

Percepción respecto al trabajo de los auditores encargados frente al cumplimiento de manuales: Los datos indicaron una percepción similar respecto al desenvolvimiento de los auditores encargados de cumplir con los manuales.

Correlación entre percepción del auditor encargado de cumplir con diligencia y la aplicación apropiada de los manuales: Los resultados entre auditor encargado de cumplir con diligencia la aplicación apropiada de los manuales de auditoría denota una correlación positiva débil (rho $=$ 0.622). En el caso del auditor encargado del manual $1, \mathrm{y}$ una correlación positiva muy débil con el auditor encargado del manual $2($ rho $=0.242)$.

\section{AUDITORES ENCARGADOS DIMENSIÓN: CUMPLIMIENTO DE LOS PROGRAMAS}

La percepción de los datos de la mediana y la media indican acción similar respecto al desenvolvimiento de los auditores encargados de cumplir con los programas; en el caso del auditor encargado del programa 3 , presenta una puntuación casi perfecta.

Correlación entre percepción del auditor encargado de cumplir con diligencia y los auditores encargados de cumplir con los programas: Se observó que en todos los casos existe una correlación positiva muy débil; y en el caso del auditor encargado de programas 2 , prácticamente nula (0.011), lo que denota muy poca congruencia entre las apreciaciones del auditor encargado de realizar con diligencia los programas o procedimientos de auditoría preestablecidos.

\section{AUDITORES ENCARGADOS DIMENSIÓN: ASPECTOS SUBJETIVOS}

Percepción del trabajo de los auditores encargados con respecto a los aspectos subjetivos: Los datos indican una percepción similar respecto al desenvolvimiento de los auditores encargados de los aspectos subjetivos.

Correlación entre percepción de los auditores encargados del cumplimiento con diligencia con respecto a los aspectos subjetivos

El coeficiente de correlación de Spearman calculado para medir el grado de asociación entre la percepción del auditor encargado de cumplir con diligencia sus labores y las variables con respecto a los aspectos subjetivos es negativo muy débil en dos casos (rho $=-0.069$ con subjetivo $1 \mathrm{y}-0.052$ con el subjetivo 2 , mientras que es positivo muy débil con el subjetivo 3 (rho $=0.215$ ).

\section{RESUMEN DEL PROCESO (supervisores)}

El modelo Alfa de Cronbach asume que la escala está compuesta por elementos homogéneos aleatoriamente seleccionados de la población de los posibles elementos que miden la misma característica; en el caso de los supervisores y sus variables tratadas, existe una fiabilidad que determinamos como óptima, equivalente a 0.970 , para un número de 13 ítem, incluida la variable diligencia.

\section{DIMENSIÓN: PAPEL DE TRABAJO}

En la percepción se presentan características muy similares entre sí, hecho que se refleja en las encuestas (media 4.28, mediana 4.00).

Correlación entre percepción del auditor supervisor como resultados de los trabajos y los papeles que los sustentan: Las correlaciones son, en todos los casos, positivas fuertes, lo cual denota bastante concordancia en las apreciaciones que se correlacionan.

\section{DIMENSIÓN: EVIDENCIA}

La percepción en el trabajo de los grupos encuestados presentan similitudes, con leves diferencias (media: 4.35; 4.25; 4.25; 4.23; 4.28, mientras la mediana 4.00 para todas). 


\section{Correlación entre percepción del auditor} supervisor como resultados de los trabajos y evidencias que los sustentan: En el conjunto de las encuestas en todos los casos positivas; son además fuertes cuando relacionan la variable supervisor trabajo con la de los grupos 1, 3, 4 y 5 , de evidencia (preguntas del cuestionario), lo cual denota bastante concordancia en las apreciaciones que se correlacionan. La correlación es muy débil en el caso del grupo 2, que indica poca concordancia entre las variables correlacionadas.

\section{DIMENSIÓN: INFORME DE AUDITORÍA}

La percepción estadística de las encuestas es bastante similar a la anterior dimensión (4.25; $4.25 ; 4.30 ; 4.28$ para la media y 4.00 para la media en general).

Correlación entre percepción del auditor supervisor como resultados de los trabajos e informes de auditoría, como resultado de sus labores: en todos los casos son positivas; además son fuertes, denotando bastante concordancia entre la variable del supervisor de trabajo y los cuatro grupos (variables) $(0.808 ; 0.8058 ; 0.809 ; 0.827)$.

\section{DISCUSIÓN \\ ANÁLISIS DE LOS DATOS: DIRECTIVOS}

ESTADÍSTICO DE FIABILIDAD (directivos): El modelo Alfa de Cronbach asume que la escala está compuesta por elementos homogéneos aleatoriamente seleccionados de la población, de los posibles elementos que miden la misma característica; en el caso de los directivos, en sus variables tratadas existe una fiabilidad, la que determinamos como aceptable, equivalente a 0.691, para un número de 44 ítem.

\section{ESTADÍSTICOS DE CAJA.- DIMENSIONES ESTIMADAS}

Los resultados nos muestran la existencia de una empatía, desde el punto de vista de los directivos hacia los supervisores, fundamentados en la percepción sobrestimada, la actitud de servicio oportuno y la atención esmerada en los trabajos, así como en su amabilidad y cortesía.

\section{CONCLUSIONES}

1. Existe falta de diligencia en las labores de auditoría, al comprobarse el incumplimiento en la aplicabilidad de las normas técnicas de auditoría, a pesar de estar de acuerdo en capacitarse periódicamente. Asimismo, además de la falta de manuales de auditoría, su entrega oportuna, hay muy poco interés frente a la falta de metodologías y técnicas, así como a la aceptación de asesoramientos por parte de la entidad auditora; más aún, ante situaciones complejas que se presentan durante el trabajo, es muy limitada la utilización de un criterio propio. Por lo expresado y fundamentándonos en los resultados de aspectos subjetivos, concluimos en apreciar debilidades en la formación personal, y profesional del auditor encargado.

2. La eficiencia del trabajo de auditoría durante el proceso, la culminación y la elaboración del informe final obedecen a diversas y posibles actitudes que pudiera asumir el supervisor, responsable del trabajo, como: regresar a completar información, coordinar con el auditor encargado para solucionar problemas de información o no darse por enterado, dependerá del grado de responsabilidad, al lograr cumplir cada objetivo en el proceso de trabajo en equipo, de tal forma que asegure un servicio de alto nivel; considerando lo concluido a nivel de los auditores encargados, se comprueba que la falta de documentación en la sustentación de los hallazgos, la necesidad de un apropiado control de calidad y la preexistencia de un sistema de trabajo contribuyen al deterioro de la eficiencia del mismo.

3. Asimismo, la evidencia insuficiente y la falta de información sobre hechos conocidos contribuyen a la deficiencia de los trabajos de auditoría; para ello, la necesidad de la exigencia de que la información justificadora sea completa y satisfactoria en su contenido; el supervisor complementa la necesidad de postergar la firma de algún in- 
forme por la falta de información suficiente. No debemos descartar que algunas causas obedecen a la falta de información que obedecerían generalmente al desconocimiento, la presión de terceros, la desactualización, o a la falta de previsión.

4. Se ha comprobado que el supervisor es consciente, en su trabajo, del cumplimiento de normas, con respecto a la confección y sustento de un informe de auditoría. Y si estas han sido observadas consistentemente, que el informe se encuentre sustentado a base de revelaciones suficientes, debidamente objetivas y respaldadas en los papeles de trabajo. También es consciente de que los informes de auditoría pudieran ser mal evaluados y/o interpretados, cuyas razones pudieran ser: falta de claridad en el trabajo, trabajos incompletos, falta de evidencia, dando lugar a la mala formulación de las conclusiones del informe de auditoría; asimismo, la falta de congruencia entre el contenido del informe final y las conclusiones pudieran dar lugar a: factores de presión, conclusiones mal formuladas, como también apreciación particular de la entidad auditora.

5. Si el punto de partida de toda gestión, para obtener un servicio de calidad, consiste en captar las exigencias de los clientes, en este caso, directivos, y analizar la forma de ofrecerles soluciones que respondan a sus necesidades dentro de las normas vigentes. En el análisis de calidad, comprobamos la conformidad que existe en expectativa y la percepción del directivo, en cuanto al trabajo exigido al equipo de auditoría, como es también la aceptación de informe, el apoyo de muebles y equipos, expresando una fiabilidad conforme, aunque se sobreestima hacia una inferencia en cuanto a la percepción esperada. Realmente los directivos están conformes solo con respecto al grado de cumplimiento en la entrega de los informes de auditoría.

6. Comprobamos que los señores directivos poseen criterios sobrestimados del trabajo muy propios de los auditores; ejemplo: procedimientos, metodologías, sistemas de trabajo de auditoría.

7. En cuanto a la experiencia laboral de los supervisores, confianza en la probidad de los informes, consideraciones ante el alto grado de riesgo de opinión de los supervisores, y la confianza en los informes de los auditores; el directivo expresa un alto grado de conformidad en cuanto a seguridad esperada.

8. Existe una apropiada empatía, desde el punto de vista del directivo hacia el supervisor, en entrevistas y presentación de los informes, fundamentada por poseer una percepción sobrestimada sobre actitud en la entrega de un servicio oportuno, atención esmerada en el trabajo de los supervisores, amabilidad y cortesía de los mismos.

\section{RECOMENDACIONES}

1. Para lograr mantener y desarrollar la diligencia del auditor, sería necesario:

- Que la responsabilidad del auditor deba interponerse como la obligación que asume un contador o administrador, miembros de una profesión, al suscribir o firmar un informe de auditoría, en cuya información deban confiar terceras personas.

- Esta obligación moral tiene que ver principalmente con la ética, la que se sustenta en una conducta basada en ciertas normas de honestidad personal en los profesionales para con la comunidad.

- No obstante, está responsabilidad profesional no lo exime de aspectos legales vinculados con el ejercicio de su profesión.

- Las empresas, las sociedades de auditoría, los colegios profesionales, como las universidades, deben coadyuvar en la formación y desarrollo del profesional en la especialidad de auditoría, mediante entrenamiento, capacitación, cursos de extensión y especialización orientados a 
niveles de experiencia: inicial, intermedia y avanzada, como también en la elaboración y renovación periódica de currículas y sillabus de los cursos de auditoría.

2. Es conveniente que el colegio de administradores, el de contadores públicos del Perú y la Contraloría General de la República ejerzan en forma obligatoria la supervisión y control en cuanto a la capacitación y formación profesional que dé grados de garantía en el desarrollo de los trabajos de auditoría. Esto permitiría:

- Inculcar, respetar y preservar la independencia e integridad de los auditores.

- Cumplir estrictamente con las normas internacionales de auditoría generalmente aceptadas, como de las normas de control interno y demás disposiciones legales en el marco de los trabajos de auditoría.

- Incrementar la solvencia moral de auditores.

3. Para contribuir resultados en cuanto a eficiencia en los trabajos de auditoría, se debe promover:

- Las entidades empresariales, como las sociedades de auditoría, deben fomentar, inculcar y defender la independencia de criterio. Menoscabarla o perderla contribuiría a perder garantías de objetividad y verdad del trabajo de auditoría y sus resultados serían total o parcialmente sesgados a la realidad.

- Las entidades empresariales, como las sociedades de auditoría, deben obligarse a cumplir las normas internacionales de auditoría, así como otras que demanden su responsabilidad profesional.

- Los auditores en todos y cada uno de sus niveles de preparación y experiencia, dado el grado de responsabilidad profesional en el proceso y resultado del trabajo, deberán:

- Evaluar a las entidades empresariales, así como a las sociedades de auditoría, antes de asumir el grado de responsabilidad y dependencia de servicio.
- Los auditores, en casos de sentir quebradas las normas que fundamentan su labor de auditoría, deben tener la suficiente fuerza moral de abstenerse en seguir con los procesos de evaluación y tener el valor suficiente de renunciar.

\section{REFERENCIAS BIBLIOGRÁFICAS}

CRONBACH, L. (1951). Coefficient Alpha and the Internal Structure of Tests. Psycometrika, 16, (Septiembre), pp. 297-334.

DÍAZ, D. 2003. Centro de competitividad. Documento de trabajo. Validación de una escala de medida para la determinación de la calidad de servicio en una institución de educación superior. [en línea]: Documento electrónico, fuente en Internet. 2003[fecha de consulta 5 de Diciembre 2003]. Disponible en: http://www.calidad.org/public/ bakolds/0993696345digene.htm.

FERNÁNDEZ, M. 2000.Validación de SERVQUAL como instrumento de medida de la calidad de servicio bancario. Revista Europea de Dirección y Economía de la Empresa, Vol. 9, N. ${ }^{\circ} 1$, pp. 57-70.

PARASURAMAN, A.; ZEITHAML, V.A. y BERRY, .L 1985. A Conceptual Model of Service Quality and Its Implications for Future Research. Journal of Marketing. pp 41-50.

RUIZ, C. 2001. Gestión de la calidad del servicio, [en línea] documento electrónico, fuente en Internet. 2003. Control de la Gestión. [Fecha de consulta 19 de Abril 2003.]. Disponible en:<http://www.5campus.com/ leccion/calidadserv $>$.

VILLAFAÑA, R. 2001. Evaluación de la calidad de los servicios SERVQUAL [en línea] documento electrónico, fuente en Internet. 2003. Universidad de las Américas Puebla México fecha de consulta 19 de Abril 2003. Disponible en: < http://mailweb.udlap.mx/ rvillafa/servqual.htm>

VISOUTA B 1998. Análisis estadísticos con SPSS para Windows. Pp Capitulo 7. pp125-133. 